\title{
A new design of photonic transmitter for terahertz spectroscopy and imaging applications
}

\author{
Moumane', J. Zbitou ${ }^{2}$, M. Latrach ${ }^{3}$, A. Errkik ${ }^{4}$, O. Chakkor ${ }^{5}$ \\ ${ }_{1,2,4}$ LMEET, FSTS Hassan 1st, University of Settat Morocco, Morocco \\ ${ }^{3}$ Microwave Group ESEO, Angers, France \\ ${ }^{5}$ Ensa of Tetuan, Morocco
}

\section{Article Info \\ Article history: \\ Received Aug 16, 2018 \\ Revised Dec 05, 2018 \\ Accepted Dec 31, 2018}

\section{Keywords:}

CPW technology

$\mathrm{CW} \mathrm{THz}$ photonic transmitter

GaAS substrate

Low-pass filter

Photodetector

\begin{abstract}
In this paper we present the Design of Continuous Wave Terahertz photonic transmitter based on photodetector, $\mathrm{THz}$ antenna, and low-pass filter (LPF) and Direct Current "DC" Probe Bias. Before validating the whole system, we processed firstly with the optimization of the antenna which is responsible of the transmitting the RF signal providing from the photodetector then we have conducted a study on the design of a low pass filter "LPF" whose role is to block the received RF signal to reach the DC probe. After the optimisation of the proposed LPF, we have integrated the different components mounted on multi-layers GaAs substrate and simulated the final photonic transmitter by using an EM solver "Momentum" integrated in ADS "Advanced Design System". The dimensions of the whole circuit are $704.99 \times 154.99 \mu^{2}$ .The simulation results permit to validate the final circuit at $1.6 \mathrm{THz}$, the proposed photonic transmitter is suitable for terahertz spectroscopy and imaging applications.
\end{abstract}

Copyright (C) 2019 Institute of Advanced Engineering and Science. All rights reserved.

\section{Corresponding Author:}

Moumane,

LMEET,

University of Settat Morocco,

50 Rue Ibnou Lhaytham B.P. 577, Settat 26002, Morocco.

Email:moumaneibti@gmail.com

\section{INTRODUCTION}

The development of the modern Femtosecond Lasers and High-Speed Photodetectors gives the opportunity to the THz domain to extend their application area.

The $\mathrm{THz}$ waves present several advantages based on interactivity with the material where it spreads and fast absorption by the atmosphere [1]. This makes it suitable for a variety of domains such as biomedical imaging, spectroscopy, security and telecommunications.

The difficulty of this domain resides in the generation, and detection of THz waves. Many methods are proposed for $\mathrm{THz}$ generation but the most used is the one relays on the coplanar waveguide (CPW) photonic transmitters [2]. The CPW technology offers in fact several advantages due to its features, like low radiation, low dispersion, easy of shunts and series connections [3]. There is another technology than CPW named Mictrostrip, which was disregard due to its high losses [4].

Compared with other methods to generate $\mathrm{THz}$ radiation such as quantum cascade lasers [5], Gunn diodes [6] or microwave multipliers [2], photonic transmitters have the advantages of simplicity, tunable $\mathrm{THz}$ wavelength and integrability with other optoelectronic devices to become compact $\mathrm{THz}$ sources [7]. This paper presents a new topology of a Continous Wave "CW" $\mathrm{THz}$ photonic transmitter composed from a photodetector associated to a large band $\mathrm{THz}$ antenna inserts in series with a low pass filter and a DC Probe Bias with a high frequency at $1.6 \mathrm{THz}$. The following sections will describe and discuss the design of the different parts of the final photonic transmitter. 


\section{PHOTONIC TRANSMITTER SYSTEM}

\subsection{THz technology}

Terahertz frequency $(\mathrm{THz})$ band is coarsely defined as a portion of the electromagnetic spectrum, which extends from 0.1 to $10 \mathrm{THz}$ and occupies an extremely large regime of the electromagnetic spectrum between the infrared and microwave bands [8]. Position of $\mathrm{THz}$ band between the microwave and infrared regime of electromagnetic spectrum as shown in Figure 1.

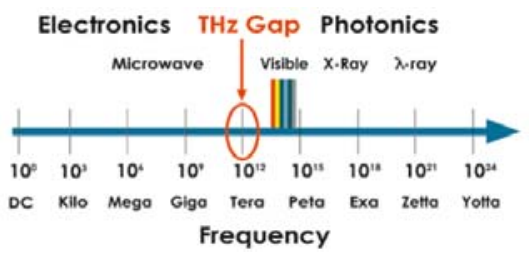

Figure 1. Position of THz band between the microwave and infrared regime of electromagnetic spectrum

\subsection{Photodetector "PD"}

A PD is a sensor that converts an optical power into an electrical current. To generate electron-holepairs, the photon energy provides from the light absorbed in a PD must be at last equal to the bandgap energy $\mathrm{Eg}$ of the absorber material [9]. This available energy of one photon is enough to excite an electron from the valence band (V.B.) to the conduction band (C.B.). For this band-to-band transition, the upper wavelength limit for photon absorption is given by [9]:

$$
\lambda_{\mathrm{g}}[\mu \mathrm{m}]=\frac{1.24}{\mathrm{E}_{\mathrm{g}}[\mathrm{eV}]}
$$

A PD has different proprieties such as:

Sensitivity: The ability of the photodiode to transform light absorbed into an electrical current in other term the number of charge carrier pairs generated per incident photon [9].

$$
\eta_{\text {ext }}=\frac{I_{P d}}{q} \cdot \frac{h v}{P_{\text {opt }}}
$$

Responsivity: where Ipd is the photogenerated current by the absorption of the optical input power Popt at a frequency $v$ mentied in (2). A common figure of merit is the external responsivity $\mathrm{R}$, defined as the ratio of photocurrent to the input optical power [9]:

$$
\mathrm{R}=\frac{\mathrm{I}_{\mathrm{Pd}}}{\mathrm{P}_{\mathrm{opt}}}=\frac{\eta_{\mathrm{ext}} \lambda[\mu \mathrm{m}]}{1.24} \mathrm{~A} / \mathrm{W}
$$

In this study we have chosen the Metal Semiconductor-Metal Travelling wave Photodetector (MSM-TPD) due to its high power-bandwidth and coplanar-waveguide fed slot owing to its easy connection with planar devices [10]. The PD based on GaAs substrate which characterized by a succession of layers as mentioned in the Figure 2 [11]:

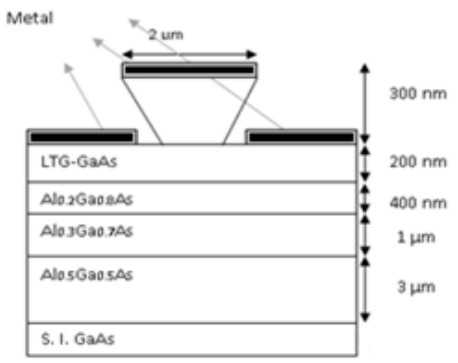

Figure 2. Structure of the photodetector based on GaAs substrate [11] 


\subsection{THz antenna design}

The presence of the antenna [12], [13] in the system CW photonic transmitter is mandatory. It's responsible of transmitting the RF signal providing from the MSM-TWPD to the LPF.

In this work we have designed a CPW triangular antenna based on the multilayer substrate presented in Figure 2. The proposed antenna was obtained by following a series of optimization methods integrated in ADS. The final dimensions are depicted in Figure 3. The final optimized parameters of the proposed CPW $\mathrm{THz}$ antenna as shown in Table 1.

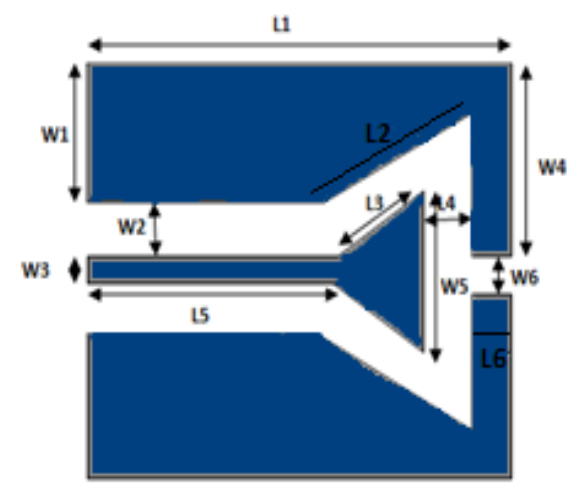

Figure 3. The THz proposed CPW triangular antenna

Table 1. The final optimized parameters of the proposed CPW THz antenna

\begin{tabular}{cc}
\hline Dimensions & Values $(\mu \mathrm{m})$ \\
\hline L1 & 75.25 \\
L2 & 42.99 \\
L3 & 35.92 \\
L4 & 9.07 \\
L5 & 28.81 \\
L6 & 6.40 \\
W1 & 27.48 \\
W2 & 10.67 \\
W3 & 5.87 \\
W4 & 38.42 \\
W5 & 41.89 \\
W6 & 6.40 \\
\hline
\end{tabular}

As shown in Figure 4, the proposed THz antenna presents a good matching impedance below -10dB [14] in the bandwidth between $1.73 \mathrm{THz}$ and $1.9 \mathrm{THz}$.

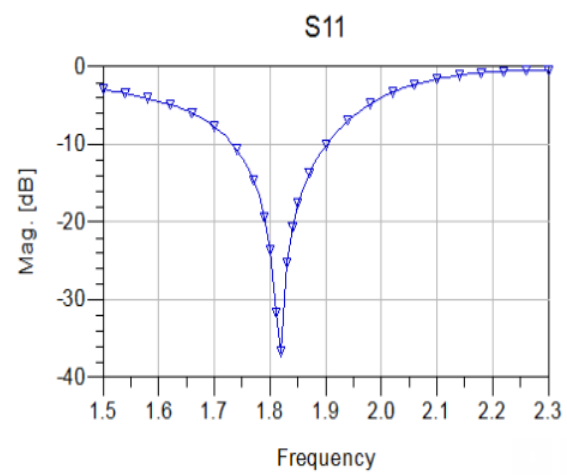

Figure 4. Reflection coefficient versus frequency of triangular CPW antenna 
To have an idea about the radiation pattern the of the proposed antenna, Figure 5 shows that the proposed antenna has bidirectional radiation at $1.83 \mathrm{THz}$ which is due to the use a CPW without an underground plane. This behavior can be interesting for $\mathrm{THz}$ imaging applications.

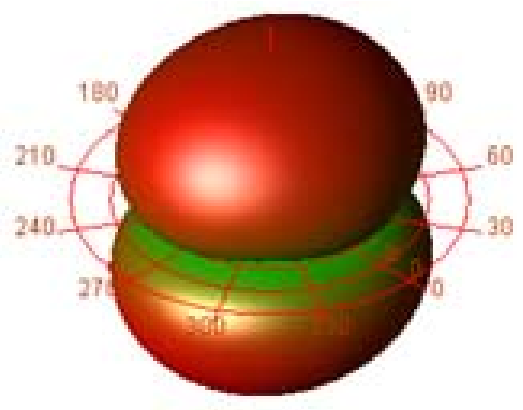

Figure 5. The $3 \mathrm{D}$ radiation pattern at $1.83 \mathrm{THz}$

To evaluate the performances of the proposed final antenna we have done a comparison between the proposed antenna and another $\mathrm{THz}$ antennas validated in literature Table 2 presents the difference in term of (dimensions, frequency bandwidth) between the proposed antenna and two other structures:

Table 2. Comparison of the antenna structures

\begin{tabular}{ccc}
\hline Antenna Structure & Length & Frequency Bandwidth \\
\hline Proposed Antenna & $75.25 \mu \mathrm{m}$ & {$[1.73 \mathrm{THz}, 1.9 \mathrm{THz}]$} \\
Antenna [15] & $172.24 \mu \mathrm{m}$ & {$[1.98 \mathrm{Thz}, 2.02 \mathrm{THz}]$} \\
Antenna [16] & $330 \mu \mathrm{m}$ & {$[1 \mathrm{THz}, 1.25 \mathrm{THz}]$} \\
Antenna [7] & $200 \mu \mathrm{m}$ & Narrow band at $650 \mathrm{GHz}$ \\
Antenna [17] & $1040 \mu \mathrm{m}$ & Narrow bandat $850 \mathrm{GHz}$ \\
\hline
\end{tabular}

As shown in this Table 2, the proposed antenna presents good performances in term of bandwidth and length (smallest design with the higher large bandwidth).

\subsection{Low-pass filter "LPF" design}

After the validation of the proposed antenna and to avoid the RF signal to reach the DC probe Bias of the PD. We have optimized new LPF structure which has the role to separate the RF signal and the DC energy permitting the Bias of the PD.

In this study we have chosen a several periodic structures presenting in the study [18], [19] The proposed structure is composed from three-unit cells as shown in Figure 6 and dimensions are presented in Table 3.

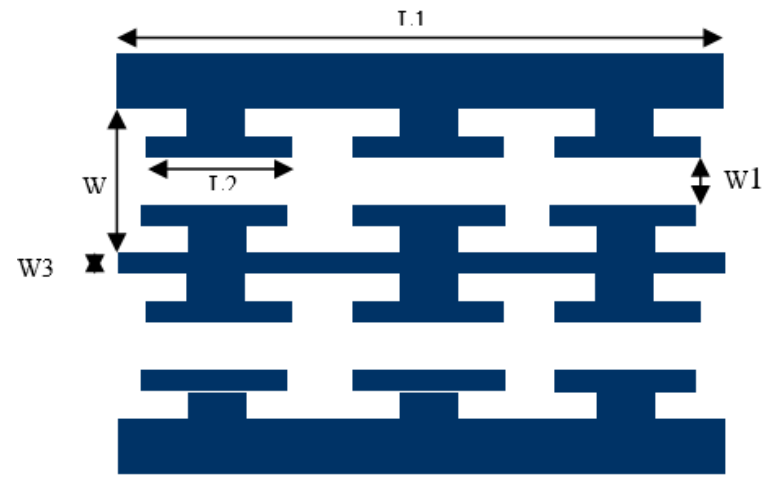

Figure 6. The layout of the proposed periodic THz LPF 
Table 3. The final optimized dimensions of the LPF

\begin{tabular}{cc}
\hline Dimensions & Values $(\mu \mathrm{m})$ \\
\hline L1 & 300.151 \\
L2 & 60.3 \\
W1 & 9.9 \\
W2 & 29.25 \\
W3 & 6.75 \\
\hline
\end{tabular}

To achieve a wide rejection band as shown in Figure 7 many series of optimization have been applied. The cutoff frequency is $0.44 \mathrm{THz}$. Which makes it suitable for CW photonic transmitter's applications. In the passband, we have a good matching input impedance under $-15 \mathrm{~dB}$. The phase of S21 coefficient is presented in Figure 8.

To study the behavior of the LPF structure, we have launched a simulation at $0.2 \mathrm{THz}$ and in the same time at $1 \mathrm{THz}$ in the rejection band. As shown in Figure 9 we can conclude that the filter let the signal pass from the input to the output at $0.2 \mathrm{THz}$ and block the signal at $1 \mathrm{THz}$ which is the attenuated band.

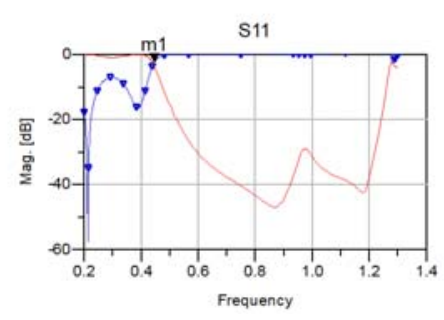

Figure 7. S-Parameters results versus frequency of the Periodic LPF

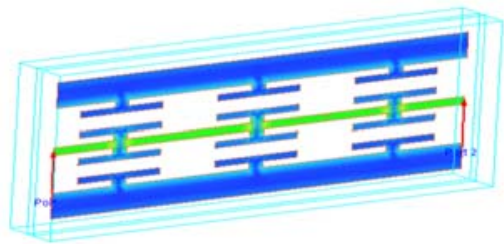

(a)

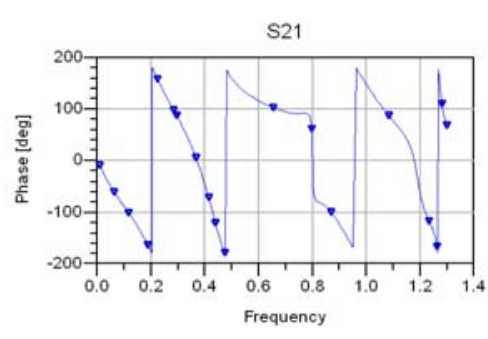

Figure 8. Phase of S21 versus frequency

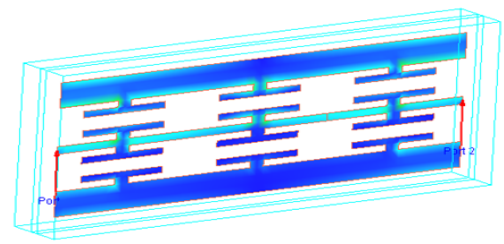

(b)

Figure 9. The current density @ (a) $0.2 \mathrm{THz}$ and (b) $1 \mathrm{THz}$

\subsection{The final structure of the photonic transmitter}

After the validation of the different components permitting the construction of the photonic transmitter. We have optimized the whole circuit which permit to validate the proposed $\mathrm{THz}$ generation system presented in the Figure 10. The different optimized parameters of the photonic transmitter as shown in Table 4.

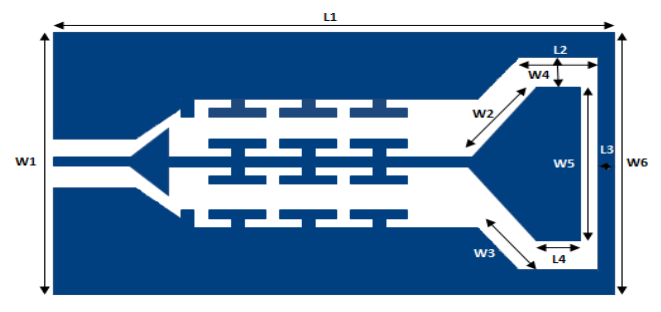

Figure 10. The proposed CW photonic transmitter 
Table 4. The different optimized parameters of the photonic transmitter

\begin{tabular}{cc}
\hline Dimensions & Values $(\mu \mathrm{m})$ \\
\hline L1 & 704.99 \\
L2 & 103.62 \\
L3 & 8.85 \\
L4 & 80.59 \\
W1 & 158.53 \\
W2 & 66.43 \\
W3 & 55.91 \\
W4 & 17.71 \\
W5 & 103.62 \\
W6 & 138.16 \\
\hline
\end{tabular}

As illustrated in Figure 11, the final $\mathrm{CW}$ THz photonic transmitter validated at $1.6 \mathrm{THz}$ with a good matching input impedance below $-15 \mathrm{~dB}$. The final circuit was optimized considered the input impedance of the $\mathrm{PD}$ which is equal to $30 \mathrm{Ohm}$.

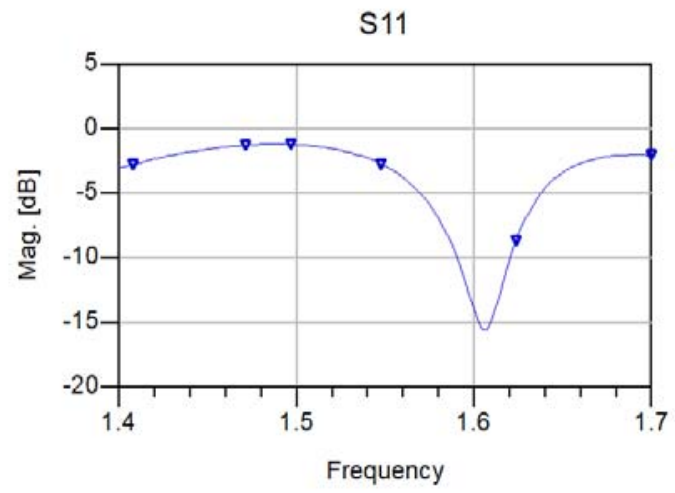

Figure 11. Simulation S-Parameters results versus frequency with $30 \Omega$

The Table 5 presents a comparison between the proposed CW THz photonic transmitter and others existing systems in term of resonant frequency:

Table 5. Comparison of the CW THz photonic transmitter systems

\begin{tabular}{cc}
\hline CW THz photonic transmitter system & Resonant frequency \\
\hline Proposed system & $1.6 \mathrm{THz}$ \\
System [7] & Narrow band at $645 \mathrm{GHz}$ \\
System [11] & Narrow band at $600 \mathrm{GHz}$ \\
\hline
\end{tabular}

\section{CONCLUSION}

The target of this paper is the conception and validation of $\mathrm{CW}$ photonic transmitter using for the generation of $\mathrm{THz}$ waves and based on $\mathrm{CPW}$ technology. The proposed system is composed from photodetector which convert the optical power to electrical signal, antenna responsible of receiving and transmitting the RF signal providing from PD, LPF using for blocking the RF signal to reach the DC probe which is the final part using for polarization. In the first stage we have used the photodetector MSM-TPWD after we moved to the validation of $\mathrm{THz}$ antenna based on multilayers GaAs substrate and "ADS" in large bandwidth $[1.73 \mathrm{THz}, 1.9 \mathrm{THz}]$. Then we optimized the "LPF" circuit with a wide rejection band from cutoff frequency $0.44 \mathrm{THz}$ until $1.23 \mathrm{THz}$. Finally, we had associated all structures to obtain the $\mathrm{CW}$ photonic transmitter with resonant frequency $1.6 \mathrm{THz}$ suitable for $\mathrm{THz}$ spectroscopy and imaging applications using an electromagnetic solver Momentum integrated in ADS. The final circuit is mounted on a multilayers GaAs substrate and having an area around $704.99 \times 154.99 \mu \mathrm{m}^{2}$ 


\section{REFERENCES}

[1] Peter H. Siegel, "Terahertz Technology," IEEE transactions on microwave theory and techniques, vol. 50(3), pp. 913, Mar 2002.

[2] J. Zbitou, C. Minot, X. Begaud, and B.Huyart, "Bow-tie Wideband Antenna Design for CW THz Photonic Transmitters," Progress In Electromagnetics Research Symposium, Cambridge, USA, pp. 431, Jul 2008.

[3] S. Khireddine, M. Drissi, R. Soares. "Flat Group Delay Low Pass Filters Using Two CPW Topologie," IEEE, pp. 2215, 2005.

[4] Emilien Peytavit, "Génération et Propagation aux fréquences Terahertz. University of sciences and technology," pp. 105, Lille France. 2002.

[5] Y. P. Gousev, I. V. Altukhov, K. A. Korolev, V. P. Sinis, M. S. Kagan, E. E. Haller, M. A. Odnoblyudov, I. N. Yassievich, K.-A. Chao, "Widely tunable continuous-wave THz laser," Appl.Phys.Lett. vol.75, pp 757-759, 1999.

[6] H. Eisele, A. Rydberg, G. I. Haddad. "Recent advances in the performance of InP Gunn devices and GaAs TUNNET diodes for the 100-300-GHz frequency range and above," IEEE Trans. Microwave Theory Tech, vol. 48, pp. 626-631, Apr 2000.

[7] Ming-Chun Tien, Hsu-Hao Chang, Ja-Yu Lu, Li-Jin Chen, Shih-Yuan Chen, Ruey-Beei Wu, Senior Member, IEEE,Wei-Sheng Liu, Jen-Inn Chyi, Senior Member, IEEE, and Chi-Kuang Sun, Senior Member, IEEE, "Device Saturation Behavior of Submillimeter-Wave Membrane Photonic Transmitters," IEEE, pp. 331.

[8] Kumud Ranjan Jha and Ghanshyam Singh, "Terahertz Planar Antennas for Next Generation Communication," International Publishing Switzerland Springer, vol. 1, 2014.

[9] Vorgelegt von, Diplom-Physiker, Andreas Beling, aus Bonn, "Periodic Travelling Wave Photodetectors with Serial and Parallel Optical Feed Based on InP", Berlin, pp. 11-13, 2006.

[10] Shi, J.-W., K.-G. Gan, Y.-J. Chiu, Y.-H. Chen, C.-K. Sun, Y.-J. Yang, and J. E. Bowers, "Metal-semiconductormetal traveling-wave photodetectors," IEEE Photonics Technology Let-ters, vol. 13(6), pp. 623-625, Jun 2001.

[11] Chi-KuangSun, "THz Optoelectronics," UltraFastOptics Laboratory (UFO) Graduate Institute of Electro-Optical Engineering and Department of Electrical Engineering National Taiwan University Taipei, TAIWAN, vol. 18.

[12] Zakaria Er-Reguig, Hassan Ammor. A Multi-element Microstrip Antenna for LTE Bands, Wi-Fi and WiMAX Application in Femtocell Network. IJEEI. vol 6(2), Jun 2018.

[13] Islam Md Rafiqul, Waheeb S. A, Sarah Rafiq, M. S. Yasmin, M. H. Habaebi, "A 2X2 MIMO Patch Antenna for Multi-Band Applications," IJEEI, vol 5(4), Dec 2017.

[14] Odile Picon, "coll. Les antennes Théorie, conception et application. Préface de Maurice Bellanger," Dunod, Paris, vol. 98, 2009.

[15] I.Moumane, J.Zbitou, A.Erkik, Larbi El Abdellaoui, Abdelali Tajmouati, M.Latrach, "Compact Photonic Transmitter Based on Annular Ring Antenna for THz Applications," TELKOMNIKA, Oct 2018, vol 16(5), pp. 2474- 2480 .

[16] I.Moumane, J.Zbitou, A.Erkik. M.Latrach, O.Chakkor, A.Fouad, "Design of CW THz Photonic transmitter based on Low Pass-Filter and Bow-tie Wideband Antenna," IJECE, vol. 8(5), pp. 3804, Oct 2018.

[17] Yi-Chun Chen, An-Shyi Liu, Shih-Yuan Chen, Ruey-Beei Wu and Chi-Kuang Sun, "Design of Rampart Slot Array Antenna in Integrated 850GHz Photonic Transmitter," APMC2005 Proceedings IEEE, 2005, vol. 2.

[18] I.Moumane, J. Zbitou, J. Terhzaz. A. Erkik, M. Latrach. "A Low-Pass Filter Design for THz Applications," CMT2016. Tetouan. pp. 1-2, May 2016.

[19] James Sor. Yongxi Qian, Tatsuo Itoh, "Miniature Low-Loss CPW Periodic Structures for Filter Applications," IEEE Transactions on microwave theory and techniques, vol. 49(12), pp. 2336-2340, Dec 2001.

Indo. J. Elec. Eng. \& Inf, Vol. 7, No. 1, March 2019: 128 - 134 